\title{
Structural, Optical and Dielectric Studies On Pure and Doped L- Alaninium Maleate Single Crystals
}

\author{
U. Karunanithi, S. Arulmozhi and J. Madhavan* \\ Department of Physics, Loyola College, Chennai - 34
}

\begin{abstract}
Good optical quality single crystals of pure and doped L-Alaninium Maleate (LAM) crystals have been grown. The crystals have been grown by slow evaporation method at a constant temperature of $35^{\circ} \mathrm{C}$ from its aqueous solution. The grown single crystals of pure and doped LAM were characterized by employing FTIR, SHG, ICP, Dielectric study and X-ray diffraction methods.
\end{abstract}

Keywords: Dielectric, Ftir, Icp, Shg, X-Ray Diffraction

\section{Introduction}

In recent years non-linear optical (NLO) crystals with high conversion efficiencies are needed for optical second harmonic generation (SHG) because of their applications in opto-electronics and photonics $[1,2]$. They have wide applications in the field of telecommunications and optical information storage devices. Amino acid family crystals are promising materials for these applications. In recent years more NLO materials with better properties have been identified and studied. The applications of NLO materials are high speed information processing, optical communications, and optical data storage. These applications depend on the properties such as transparency, birefringence, refractive index, dielectric constant and thermal, photochemical and chemical stability. Among the organic NLO materials, $\alpha$ amino acids exhibit the properties like molecular chirality and weak Vander Waals and hydrogen bonds. Amino acid L alanine is an efficient organic NLO compound. The $\mathrm{L}$ alanine molecule $\mathrm{C}_{3} \mathrm{H}_{8} \mathrm{NO}_{2}{ }^{+}$exists in the cationic form with a positively charged amino group. Its crystal structure was determined by both X-ray and neutron diffraction analyses [3-4]. The growth and characterization of $\mathrm{L}$ alanine crystals were reported by many authors earlier [5-7]. The second harmonic generation efficiency of $\mathrm{L}$ alanine is one third of the standard Potassium Hydrogen Phosphate (KDP) [8]. It is also used as radiation dosimetry in food industries [9]. $\mathrm{L}$ alaninium maleate $\left(\mathrm{C}_{3} \mathrm{H}_{8} \mathrm{NO}_{2}{ }^{+} \mathrm{C}_{4} \mathrm{H}_{3} \mathrm{O}_{4}{ }^{-}\right)$is an excellent organic NLO crystal. L alaninium maleate (LAM) crystallizes in $\mathrm{P} 22_{1} 2_{1} 2_{1}$ space group. In the present work pure and doped LAM crystals were grown. The dopants are $\mathrm{La}^{3+}$ and $\mathrm{Nd}^{3+}$. The cell parameter values of the doped crystals were found to be slightly altered. The addition of dopant has improved the optical properties. The nature of constituents and functional groups present in the crystals are confirmed by FT-IR studies. The microhardness studies and dielectric studies of both pure and doped LAM crystals were carried out. The NLO property of pure and doped LAM crystals was studied using Kurtz second harmonic generation test. The samples were illuminated using Q switched mode locked Nd:YAG laser. The emission of green radiation from the crystals confirmed the second harmonic signal generation.

\section{Experimental Procedure}

Analytical grade L-alanine (AR grade) and maleic acid was dissolved in double deionized water. In order to grow good quality crystals, it is essential to increase purity up to a respectable level. In the present study, the commercially available salt was dissolved in water and purified by the repeated recrystallisation process and the recrystallised material was used to prepare the saturated solution. The solubility of the pure and doped LAM was measured at different temperatures and the drawn solubility curves are shown in Fig 1. It is seen that the solubility of both pure and doped LAM increases with temperature. The resulting aqueous solution was filtered and allowed to evaporate under optimized conditions to grow crystals by slow evaporation method at room temperature $\left(30^{\circ} \mathrm{C}\right)$. The reaction that takes place between L-alanine and maleic acid in water medium is as follows:

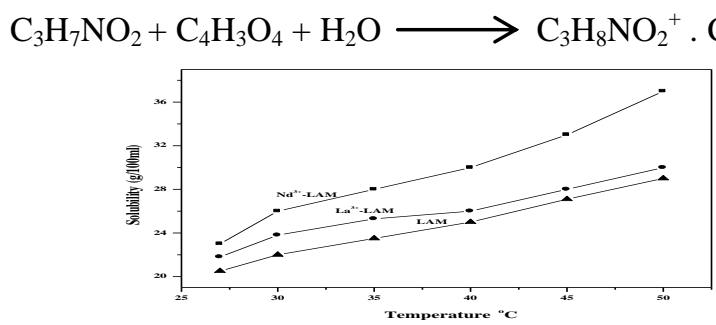

Fig 1. Solubility of LAM, $\mathrm{La}^{3+}$ - LAM, $\mathrm{Nd}^{3+}$ - LAM

www.iosrjournals.org 

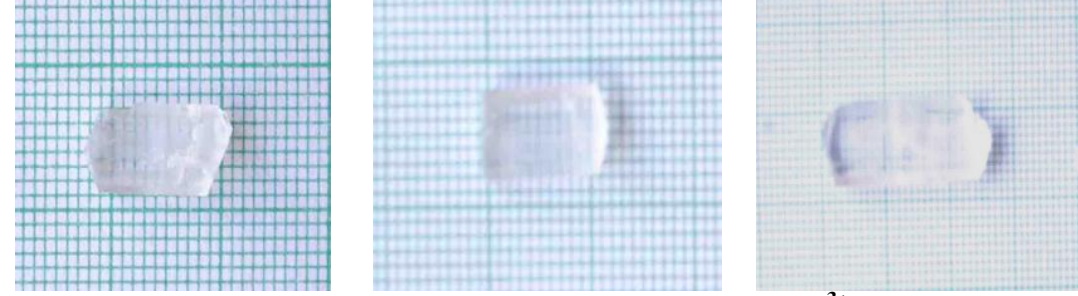

Fig 2. Photographs of the as grown (a) pure LAM, (b) $\mathrm{La}^{3+}$-LAM and $\mathrm{Nd}^{3+}$-LAM crystals

The same procedure was applied to grow the metal $\left(\mathrm{La}^{3+}\right.$ and $\left.\mathrm{Nd}^{3+}\right)$ doped crystals by adding 2 mol \% of $\mathrm{La}^{3+}$ and $\mathrm{Nd}^{3+}$ to the respective LAM solution. The growth and size of the doped crystals are respectively faster and better than the pure crystals. The incorporation of dopant into the parent solution has promoted the growth rate and improved the quality of the crystals. The pure and doped LAM crystals were grown by slow evaporation technique at room temperature. The photographs of the as grown pure and doped crystals are shown in Fig 2. Bulk crystals were grown by successive recrystallisation and purification and the crystals are found to be transparent and free from defects.

\section{Characterization}

\subsection{Powder XRD studies}

The structural properties of single crystals of pure and doped LAM have been studied by X-ray powder diffraction technique. Powder X-ray diffraction studies of pure, $\mathrm{La}^{3+}$ and $\mathrm{Nd}^{3+}$ doped LAM crystals were carried out, using Siemens D500 X-ray diffractometer with $\mathrm{Cu} \mathrm{K}_{\alpha}(\lambda=1.5406 \AA$ ) radiation. The samples were scanned for $2 \theta$ values from $10^{\circ}$ to $50^{\circ}$ at a rate of $2^{\circ} / \mathrm{min}$. Fig 3 shows the Powder XRD pattern of the pure and doped LAM crystal.

The diffraction patterns of the pure and doped LAM crystals have been indexed by least square fit method. The lattice parameter values of the pure LAM crystal has been calculated and is well matched with the
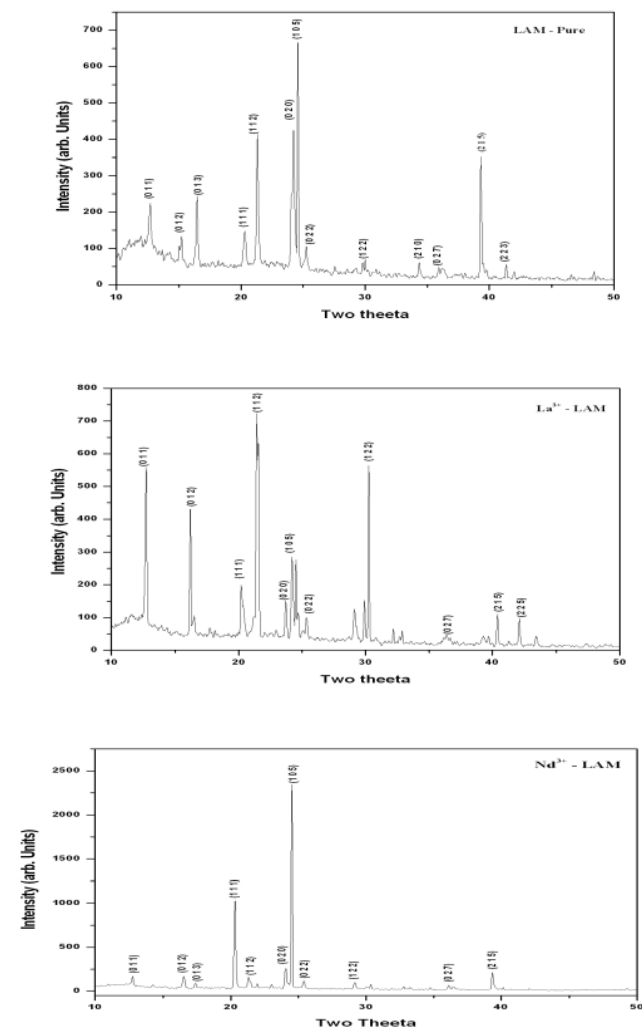

Fig 3. XRD pattern of Pure and Doped LAM single crystals

reported literature. It is seen that both the pure and doped crystals crystallizes in orthorhombic P $2{ }_{1} 2_{1} 2_{1}$ space group and the lattice parameters are shown in Table1. There are slight variations in the lattice parameters 
Structural, Optical And Dielectric Studies On Pure And Doped L-Alaninium Maleate Single Crystals and cell volume of the pure and doped crystals. These variations are due to the incorporation of $\mathrm{La}^{3+}$ and $\mathrm{Nd}^{3+}$ in the LAM crystal lattice.

Table 1. Lattice Parameters of Pure LAM, $\mathrm{La}^{3+}$ - LAM, $\mathrm{Nd}^{3+}$ - LAM

\begin{tabular}{|c|c|c|c|}
\hline $\begin{array}{c}\text { Lattice } \\
\text { parameters }\end{array}$ & Pure LAM & $\mathbf{L a}^{3+}$ - LAM & $\mathbf{N d}^{3+}$ - LAM \\
\hline $\mathrm{a}(\AA)$ & 5.574 & 5.580 & 5.584 \\
$\mathrm{~b}(\AA)$ & 7.358 & 7.371 & 7.370 \\
$\mathrm{c}(\AA)$ & 23.720 & 23.682 & 23.691 \\
\hline Crystal System & Orthorhombic & Orthorhombic & Orthorhombic \\
\hline Space group & $\mathrm{P} 22_{1} 2_{1} 2_{1}$ & $\mathrm{P} 2_{1} 2_{1} 2_{1}$ & $\mathrm{P} 2_{1} 2_{1} 2_{1}$ \\
\hline Volume $\left(\AA^{3}\right)$ & 972.84 & 974.04 & 974.98 \\
\hline
\end{tabular}

\subsection{Inductively Coupled Plasma studies}

The exact percentage of the $\mathrm{La}^{3+}$ and $\mathrm{Nd}^{3+}$ present in the respective doped crystals is determined. $10 \mathrm{mg}$ of fine powder of the $\mathrm{La}^{3+}$ and $\mathrm{Nd}^{3+}$ doped LAM crystals were dissolved in $100 \mathrm{ml}$ of triple distilled water respectively, and the prepared solutions were subjected to Inductively Coupled Plasma studies (ICP). The results shows that only $0.38 \%$ of $\mathrm{La}^{3+}$ and $0.89 \%$ of $\mathrm{Nd}^{3+}$ are present in the respective samples, out of $2 \%$ of the dopant. It is seen that the amount of dopant incorporated in to the doped crystal is less than the concentration of the dopant in the corresponding solution.

\subsection{NLO studies}

The grown crystals of pure and doped LAM were subjected to Kurtz Second Harmonic Generation (SHG) test using the Nd:YAG Q-switched laser beam for the nonlinear optical (NLO) property. The second harmonic signal of $301 \mathrm{~mW}, 382 \mathrm{~mW}$ and $405 \mathrm{~mW}$, respectively were obtained for pure, $\mathrm{La}^{3+}$ and $\mathrm{Nd}^{3+}$ doped LAM with reference to KDP $(275 \mathrm{~mW})$. Thus, the SHG efficiency of pure, $\mathrm{La}^{3+}$ and $\mathrm{Nd}^{3+}$ doped crystals is nearly $1.1,1.4$ and 1.5 times greater than KDP. It is seen that, the $\mathrm{La}^{3+}$ and $\mathrm{Nd}^{3+}$ metal dopants have increased the efficiency of pure LAM.

\subsection{FT- IR spectra}

In order to qualitatively analyze the presence of functional groups in LAM, FT-IR spectra of the pure and metal doped LAM crystals were recorded in the range $400 \mathrm{~cm}^{-1}$ to $4000 \mathrm{~cm}^{-1}$, using $\mathrm{KBr}$ pellet on BRUKKER IFS FT-IR Spectrometer. The FT-IR Spectra of both the pure and doped LAM crystals are shown in Fig 4. A broad, strong absorption in the $3300-2300 \mathrm{~cm}^{-1}$ range, including the absorptions at 3206 and $2930 \mathrm{~cm}^{-1}$ correspond to the stretching bands of the $\mathrm{NH}_{3}{ }^{+}$ion of the aminoacid. This region results from superimposed $\mathrm{O}-$ $\mathrm{H}$ and $\mathrm{NH}_{3}{ }^{+}$stretching bands. Absorption in this region is also characterized by multiple fine structures on the lower wavenumber side of the band and the weak absorptions due to $\mathrm{COO}^{-}$ions. A strong band arising from $\mathrm{C}_{-}$ $\mathrm{COO}^{-}$stretching is observed at $1219 \mathrm{~cm}^{-1}$. Further strong carbonyl absorption at $1720 \mathrm{~cm}^{-1}$ confirms the $\mathrm{COOH}$ and $\mathrm{COO}^{-}$groups of the compound. Other characteristic vibrations establishing the identity of the functional groups present in the compound are represented in Table 2. The FT-IR Spectra of both the pure and doped LAM confirm the structural aspects of pure compounds. The spectra do not show any significance change due to the addition of metal dopant into the crystal lattice.

\begin{tabular}{|c|c|c|c|}
\hline \multicolumn{3}{|c|}{ Wave number $\left(\mathrm{cm}^{-1}\right)$} & \multirow{2}{*}{ Assignments } \\
\hline Pure LAM & $\mathbf{L a}^{3+}-\mathbf{L A M}$ & $\mathrm{Nd}^{3+}-\mathrm{LAM}$ & \\
\hline 3206 & 3206 & 3206 & $\mathrm{NH}_{3}$ asymmetric stretching \\
\hline 2930 & 2920 & 2931 & C-H stretching \\
\hline 1720 & 1719 & 1719 & $\mathrm{COO}^{-}$asymmetric stretching \\
\hline 1374 & 1364 & 1374 & $\mathrm{COO}^{-}$symmetric stretching \\
\hline 1219 & 1218 & 1218 & $\mathrm{COO}^{-}$vibration \\
\hline 1107 & 1106 & 1106 & C-O stretching \\
\hline 862 & 862 & 862 & C-C stretching \\
\hline 762 & 754 & 761 & $\mathrm{CH}_{2}$ rocking \\
\hline 661 & 660 & 661 & $\mathrm{COO}^{-}$plane deformation \\
\hline 587 & 586 & 587 & $\mathrm{CO}$ \\
\hline
\end{tabular}

Table 2. FTIR Assignments of Pure LAM, $\mathrm{La}^{3+}$ - LAM \& Nd ${ }^{3+}$ - LAM 

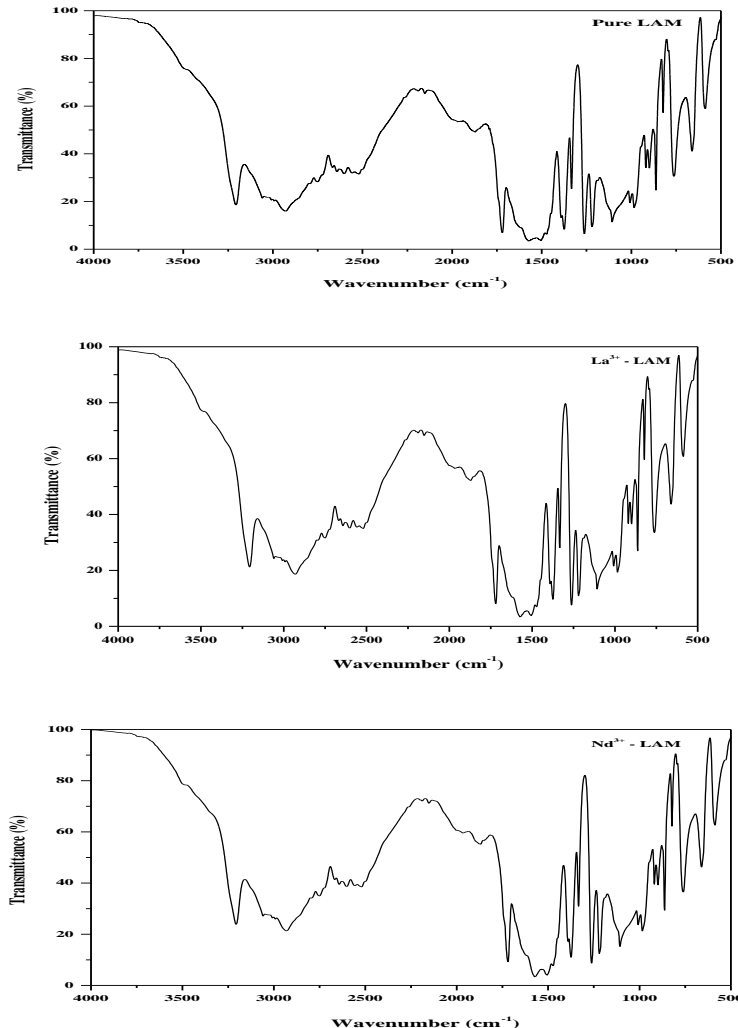

Fig 4. FTIR Spectra of Pure and Doped LAM crystals

\subsection{Microhardness studies}

The variations of Vickers hardness number with applied load for pure and doped LAM crystals are shown in Fig 5. The plot indicates that the hardness of the pure and doped LAM crystal decreases with increasing load. The decrease of the microhardness with the increasing load is in agreement with the normal indentation size effect (ISE).

By plotting $\log \mathrm{P}$ verses $\log \mathrm{d}$, the value of the work hardening coefficient (n) of pure, $\mathrm{La}^{3+}$ and $\mathrm{Nd}^{3+}$ doped LAM was found to be $1.25,1.15$ and 1.07 respectively. According to Onitsch, $1.0 \leq \mathrm{n} \leq 1.6$ for hard materials and $n>1.6$ for soft materials [1]. Hence, it is concluded that LAM is a hard material.

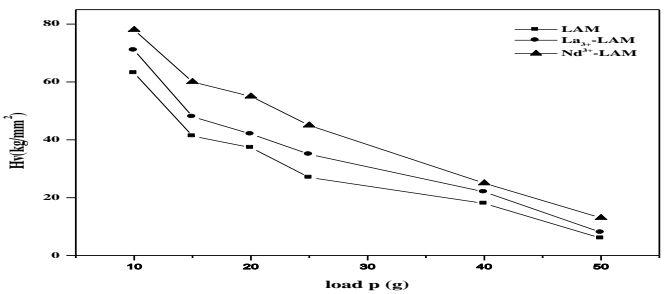

Fig 5. Variation of hardness with applied load for the plane (011)

\subsection{Dielectric studies}

The dielectric study of pure and doped LAM single crystals were carried out using the instrument, HIOKI 3532-50 LCR HITESTER. The capacitance of the sample was noted for the applied frequency that varies from $100 \mathrm{~Hz}$ to $5 \mathrm{MHz}$ at room temperature. Fig 6. shows the plot of dielectric constant $\left(\varepsilon_{\mathrm{r}}\right)$ versus $\log$ frequency for pure and doped crystals. The dielectric constant is high at low frequencies and decreases with the applied frequency. The very high value of $\varepsilon_{\mathrm{r}}$ at low frequencies may be due to the presence of all the four polarizations namely, space charge, orientational, electronic and ionic polarization and its low value at higher frequencies may be due to the loss of significance of these polarizations gradually. Displacement of an ion from an equilibrium position is equivalent to the placing of the fictitious dipole at the state with the ion in equilibrium. Although positive and negative ions displace in opposite directions in an electric field, the induced moments all have the same sign. Since ionic polarization is related to the oscillation of ions, the proper frequencies are much lower than those of electrons due to large differences between masses. The variation of dielectric loss with frequency is shown in Fig 7. 


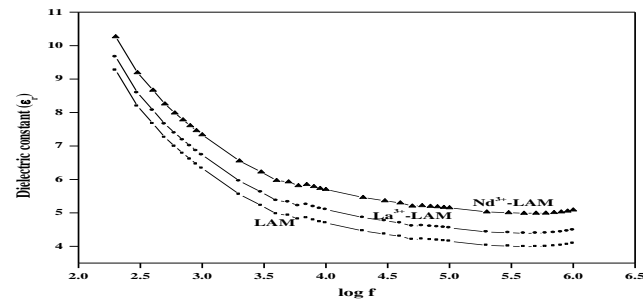

Fig 6. Plot of dielectric constant $\left(\varepsilon_{\mathrm{r}}\right)$ versus log frequency of pure LAM, $\mathrm{La}^{3+}$-LAM and $\mathrm{Nd}^{3+}$-LAM single crystals

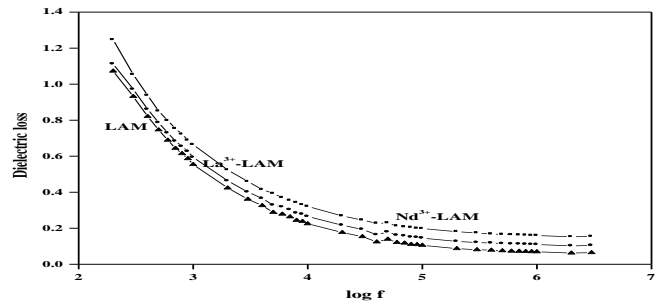

Fig 7. Plot of dielectric loss versus log frequency of pure LAM, $\mathrm{La}^{3+}-\mathrm{LAM}$ and $\mathrm{Nd}^{3+}$-LAM single crystals

The low dielectric loss with high frequency of the pure, $\mathrm{La}^{3+}$ and $\mathrm{Nd}^{3+}$ doped sample proves that these samples possess enhanced optical quality with lesser defects. This characteristic is an important parameter which is of vital importance for nonlinear optical materials in their applications [3]. The dielectric loss of pure, $\mathrm{La}^{3+}$ and $\mathrm{Nd}^{3+}$ doped LAM shows similar features. Thus, it is concluded that the dielectric nature of the pure LAM is marginally altered by the presence of metal dopant.

\section{Conclusion}

Good quality single crystals of pure, $\mathrm{La}^{3+}$ and $\mathrm{Nd}^{3+}$ doped L-Alanine Maleate (LAM) were grown successfully by slow evaporation technique. Powder X-ray diffraction studies were carried out, and the lattice parameters are calculated by least square fit method. ICP studies on the doped crystals shows that the amount of dopant incorporated in to the doped crystal is less than the concentration of the dopant in the corresponding solution. It is seen that the incorporation of dopant into the parent solution has promoted the growth rate and improved the quality of the crystals. The presence of functional groups in pure and doped LAM were analysed by FT-IR studies. NLO studies proved that the $\mathrm{La}^{3+}$ and $\mathrm{Nd}^{3+}$ metals have increased the efficiency of pure LAM. The presence of dopants has improved the Nonlinear optical (NLO) properties of the grown crystals and hence the $\mathrm{La}^{3+}$ and $\mathrm{Nd}^{3+}$ doped LAM crystals can be used as a promising material for nonlinear device fabrication.

\section{References:}

[1]. Sujatha T, Cyrac Peter A, Vimalan M, MerlineShyla J, Madhavan J., Physica B., 405(2010) 3365.

[2]. Victor Antony Raj M and Madhavan J., Archives of physics Research, 2 (1) (2011)160-168.

[3]. S. Senthil, S. Pari, R. John Xavier, J. Madhavan, Optik, 2011, 123, pp 104

[4]. G. Prabagaran, M. Victor Antony Raj, S. Arulmozhi and J. Madhavan, Der Pharma Chemica, 2011,3 (6):637

[5]. M. Victor Antony Raj, J.Madhavan, M. Gulam Mohamed, J. Comput. Method. Mol. Design, 2011, 1 (4):57-64

[6]. M.S.Lehman, T.F. Koetzle, W.C. Hamilton, J.Am.Chem.soc.94(1972)2657

[7]. L.Misoguti, A.R.Varela, F.D.Nunes, V.S.Bagnato, F.E.A. Mela, J.Mendes Filho, S.C.Zilio, Opt.Mater 6(1996)147.

[8]. S. K. Kurtz and T. T. Perry, J. Appl. Phys. 39, 3798 (1968).

[9]. G.Ramesh Kumar, S.Kokulraj, R.Sankar, R.Mohan, S.Pandi, R.Jayavel

[10]. B.Rakvin, Appl. Radiat. Isot. 47(1996)965 